\title{
Cytoskeletal Crosstalk in Cell Migration
}

Shailaja Seetharaman ${ }^{1,2}$ and Sandrine Etienne-Manneville ${ }^{1, *}$

${ }^{1}$ Cell Polarity, Migration and Cancer Unit, Institut Pasteur, UMR3691 CNRS, Equipe Labellisée Ligue Contre le Cancer, F-75015, Paris, France.

${ }^{2}$ Université Paris Descartes, Sorbonne Paris Cité, 12 Rue de l'École de Médecine, 75006 Paris, France.

*Correspondence: setienne@pasteur.fr

Keywords: cytoskeleton, actin, microtubules, intermediate filaments, focal adhesions, mechanotransduction, adhesion 


\begin{abstract}
Cell migration is a highly dynamic process driven by the cytoskeleton, which mainly comprises the actin microfilaments, microtubules and intermediate filaments. During migration, cells polarize and form protrusions at the front where new adhesions are formed. These nascent adhesions mature into focal adhesions that transmit the traction forces required for movement. All of these steps are coupled to major cytoskeletal rearrangements and are controlled by a wide array of signaling cascades. The constant crosstalk between actin, microtubules, and intermediate filaments ensures their coordinated dynamics to facilitate cell migration. Here, we first describe how master regulators, such as RhoGTPases, can simultaneously control the three cytoskeletal structures. We then summarize the recent crosstalk mechanisms by which cytoskeletal networks can locally regulate one another in order to function in a coordinated and efficient manner during migration.
\end{abstract}




\section{Cell migration: general principles and variations}

Cell migration is essential for fundamental physiological processes such as development, tissue repair and immune responses [1]. Cells migrate throughout embryonic development and in adulthood. For instance, the collective migration of cells in the blastocyst gives rise to the three germ layers, and these cells further migrate out to target sites and form tissues and organs. During wound healing and tissue renewal, migration of leukocytes to sites of infection and inflammation plays a key role in immune responses $[2,3]$. Cell migration also contributes to several pathological conditions such as chronic inflammatory diseases and cancer invasion. In this context, unravelling the mechanisms that regulate cell migration can help design therapeutic strategies [4].

The speed, directionality, persistence and modes of migration can be influenced by several factors including the cell type, the presence of neighboring cells or soluble cues, and the physical and biochemical properties of the extracellular matrix (ECM, see Glossary). Distinct cell morphologies, adhesive properties and cytoskeletal dynamics give rise to different modes of migration, such as the amoeboid and mesenchymal modes. Amoeboid migration refers to the movement of cells lacking mature focal adhesions (FAs) and stress fibers. In this case, cells undergo a contraction-based bleb migration or use actin-driven protrusions to glide on the substrate [5-9]. On the other hand, cells with strong matrix adhesions and contractility migrate in a mesenchymal manner (Figure 1), where they adhere to the ECM through integrin-mediated FAs. FAs serve as foci that transmit traction forces to pull the cell forward [10-13]. The highly adhesive cells exert higher forces on the substrate and migrate much slower than amoeboid cells with weaker adhesions (mesenchymal cells: 0.1-1 $\mu \mathrm{m} / \mathrm{min}$; amoeboid cells: $4-20 \mu \mathrm{m} / \mathrm{min}$ ). Cells can switch between the two migration modes (termed as plasticity) depending on matrix stiffness, confinement, adhesion levels, contractility, and changes in the activity of certain proteins such as the small GTPases of the Rho family $[8,14,15]$. Moreover, cells can migrate individually (e.g. neutrophils and fish keratocytes) or collectively as groups, sheets or chains (e.g. neural crests and epithelial cell sheets) [1]. In contrast to single cell migration, collective migration involves the synchronized movement of several cells connected by cell-cell contacts. 
Cell migration in 2D systems can be described as a multi-step process that involves the spatiotemporal regulation and dynamics of the cytoskeleton, which comprises the actin microfilaments, microtubules (MTs) and intermediate filaments (IFs) (Box 1, Figure 1). However, in contrast to flat 2D substrates, cells in the body experience a constantly changing ECM and have to adapt their morphologies and modes of migration accordingly. In recent years, the mechanisms of 3D cell migration (Box 2, Figure I) have been explored in order to better understand the physiological cellular process. In addition to amoeboid and mesenchymal modes of 3D migration, cells can move through an intermediary lobopodial type of migration, whereby tightly adherent cells use actomyosin contractility, hydrostatic pressure and nuclear pistoning to form bleb-like blunt protrusions called lobopodia (Box 2, Figure I) [16].

The individual role of each cytoskeletal element in cell migration has been well described (for reviews, refer to [17-19]); however, all three cytoskeletal networks must function in a coordinated manner for a cell to migrate efficiently. In addition to sharing common regulators, each cytoskeletal network can influence the others directly through cytoskeletal crosslinkers or indirectly via signaling cascades. This crosstalk between cytoskeletal structures helps coordinate their dynamics during each step of migration as well as establish feedback mechanisms that allow cells to adapt to the constantly changing microenvironment. In this review, we will focus on the recent developments demonstrating the crosstalk between actin, MTs and IFs during the establishment of cell polarity, the formation of protrusions, cell adhesion and contractility, and highlight their involvement in the feedback mechanisms that fine-tune the migratory behavior of cells.

\section{Cytoskeletal networks are controlled by common master regulators}

A straightforward manner to coordinate the dynamics of the cytoskeletal elements is through signaling cascades that can simultaneously regulate all the networks. Since their discovery in the 1980's, the Rho family of small GTPases (RhoGTPases) have been shown to orchestrate cell migration through their effects on the cytoskeleton [20]. Initially characterized by their impact on actin dynamics, RhoGTPase signaling has subsequently been linked to the regulation of MTs and IFs [21-24]. 
Ras-related C3 botulinum toxin substrate 1-3 (Rac1, Rac2 and Rac3) and Cell division cycle 42 (Cdc42) activity along with phosphoinositide signaling at the cell front promote actin polymerization at the protruding cell front, whereas Ras homolog family members $A, B$ and $C$ (RhoA, RhoB and RhoC) stabilize actin filaments and control actomyosin contractility. In addition to these extensively studied RhoGTPases, a few others have been recently described to play a role in actin dynamics and cell migration. In contrast to RhoA, RhoB and RhoC, Rnd3/RhoE and Rnd1, although cell-type dependent, inhibit Rho kinase (ROCK) and stress fiber assembly to act as Rho antagonists $[25,26]$. RhoH interacts with Rac1 and p21-activated kinase (PAK) 2, and promotes lamellipodium extension and persistence during prostate cancer cell migration, possibly through changes in the actin or MT networks [27]. Another RhoGTPase, RhoU, cooperates with PAK4 to promote cell adhesion and migration [28]. Also, RhoD regulates actin dynamics, protrusion formation and directed cell migration by binding to WASp homologue associated with actin Golgi membranes and microtubules (WHAMM) [29, 30].

With the development of optogenetic tools and Förster Resonance Energy Transfer (FRET) sensors for RhoGTPases and their regulators (Guanine nucleotide exchange factors GEFs and GTPase-activating proteins GAPs), studies have shown that the spatio-temporal regulation, including the local activation and gradients of RhoGTPases, controls cell directionality and stabilization of the protrusion [21, 31-35]. Recent systems analyses demonstrated that the GEFs and GAPs occupy different regions/organelles/compartments/structures within the cell, and can therefore regulate the local activity of RhoGTPases [33]. For instance, FA regions have a spatial segregation of several RhoGEFs and GAPs. This local segregation alters Rac1 activity near the cell periphery, suggesting that the spatio-temporal organization of the GEFs helps maintain a balance between the extension of a protrusion by Rac1 and the contractility of the cell body through RhoA during cell migration [33].

RhoGTPases, along with their GEFs and GAPs, are also involved in the control of MT dynamics, anchoring and stabilization at the cell cortex [36-39]. Cdc42 not only promotes actin polymerization and reorganization through Neural Wiskott-Aldrich syndrome protein (N-WASP) and formins, but also controls cell polarity through its effects on MT and Golgi organization, centrosome positioning or downstream of vesicular trafficking via the polarity protein Par6- 
Atypical protein kinase C (aPKC) complex [40-45]. Cdc42 also contributes to the reorganization of the IF network by regulating MT-driven transport of the filaments [46]. Thus, Cdc42 acts as a master regulator of cell polarity by simultaneously promoting the localized polymerization of actin as well as the extension and polarized distribution of MTs and IFs in order to promote the formation and maintenance of a stable membrane protrusion.

Similarly, Rho and Rac have been shown to concomitantly control actin, MTs and IFs [32, 46-50]. Activation of Rac1 facilitates lamellipodia formation through actin polymerization (via the WASP-family verprolin-homologous protein complex SCAR/WAVE [51, 52]), MT rescue (by PAK1dependent phosphorylation and inactivation of stathmin, a MT catastrophe inducing protein [53, 54]) and local disassembly of IFs (by the phosphorylation of vimentin [55]). Vimentin can be phosphorylated by ROCK, which is well-known regulator of actomyosin contractility, downstream of Rho. The levels of vimentin and the ROCK-dependent phosphorylation status of vimentin has been linked to cell migration speed, possibly through its effects on IF assembly and organization [56-58]. Rho signaling can also control migration through its effects on the MT network organization and stability [59-61]. Altogether, Rho promotes the formation of longer lived or highly stable structures such as stress fibers, stable MTs and bundled IFs that are oriented in the direction of migration, whereas Rac and Cdc42 rather increase cytoskeletal dynamics to promote membrane remodeling at the cell periphery.

Apart from the RhoGTPases, additional signaling cascades (such as phosphoinositide signaling) or proteins (such as cofilin and the tumor suppressor Adenomatous polyposis coli APC) influence the three filamentous networks [62,63]. All these master regulators control the general front-to-back coordination of the cytoskeletal structures. Interestingly, the localization and activity of these regulatory proteins are in turn controlled by the cytoskeleton, further contributing to the crosstalk between actin, MTs and IFs. Such a crosstalk allows fine-tuning of the cytoskeletal dynamics required for optimal cell migration.

\section{Collaborative polarization of the cytoskeletal networks}

The first step of cell migration involves the establishment of a front-rear polarity axis (Box 1, Figure 1), which is important for directionality and persistence during migration. The crucial role 
of MTs in mesenchymal cell polarity is well documented - the asymmetrical dynamics and regulation of MTs imparts polarity and directionality. The centrosome and Golgi apparatus, which are MT organizing centers, are frequently repositioned in front of the nucleus and promote MT growth towards the cell front (Box 1, Figure 1) [64, 65]. For instance, during epithelial-tomesenchymal transition (EMT)-associated migration, epithelial cells with the centrosome on the apical pole undergo MT destabilization-mediated polarity reversal as they transition into mesenchymal cells that invade through the tissue [66]. In micropatterned cells, centrosome positioning and MT organization was shown to be dependent on the anisotropy of the actomyosin network [67], which supports the idea that the architecture of the actomyosin network is important for the polarization of MTs. In addition to centrosomal MTs, noncentrosomal MTs can also orient themselves to promote cell polarity and directionality during migration [68]. In order to ensure cell polarity and directed cell migration, the microtubule organization must be coupled to that of actin and IFs, as the common polarized distribution of all networks is essential for the protrusion of the cell front and retraction of the rear (Box 1, Figure 1).

\subsection{Interplay between actin and MTs}

Despite the entrenched role of MTs in the polarity of different cell types, the mechanisms by which MTs influence the other cytoskeletal structures is not fully understood yet. One major impact of the orientation of the MTs is the polarization of MT-mediated intracellular transport towards the leading edge. MT-associated motors influence actin polymerization at the leading edge of the cell by carrying mRNAs of proteins that regulate the actin cytoskeleton, for example Actin-related protein 2/3 (Arp2/3) (Figure 1, 2A) [46, 69-72]. Improper localization of Arp2 mRNA away from the leading edge impairs directionality during migration [73]. It was shown that Rhoand contractility-dependent formation of detyrosinated MTs promotes the transport of APC mRNA to the cell front, suggesting a possible crosstalk mechanism by which actin and MTs drive the transport of mRNA transcripts that are essential for maintaining cell protrusions [74]. MT- 
dependent trafficking of post-Golgi carriers and recycling endosomes also plays a key role in the delivery of molecules to the leading edge of the cell. For instance, recycling of endosomes helps deliver Rac and Cdc42 to the cell front, along with their effector protein Rho guanine nucleotide exchange factor 7 (ARHGEF7/ $\beta$-PIX) (Figure 1, 2A) [75, 76], thus creating a positive feedback loop reinforcing cell polarity, stabilizing the protrusion, and promoting persistent migration.

The global polarization of the MT network influences the overall cytoskeletal reorganization. The dynamic instability observed at MT plus-ends near the cell front can finetune local actin dynamics. One major mechanism underlying the effects of MTs on the actin network is the activation of Rac1 and RhoA by MT polymerization and depolymerization respectively [77, 78], which can, in turn, influence the dynamics of the three cytoskeletal elements (See Part 1). In addition, MTs control the activity or localization of the direct regulators of actin dynamics; for instance, a protein of the WASP family, WHAMM, binds to MTs and activates Arp2/3 to promote actin polymerization [79]. Several MT plus-end tracking proteins, +TIP proteins, are tightly coupled to MT dynamics but also appear to play a role in the regulation of actin. Single molecule fluorescence microscopy studies showed that the MT +TIP, cytoplasmic linker protein of $170 \mathrm{kDa}$ (CLIP-170), tightly associates with the formin mammalian diaphanous 1 (mDia1) [80]. The End-binding protein 1 (EB1) at the plus-ends of growing MTs recruits the CLIP170-mDia1 complex to trigger actin polymerization and lamellipodia formation (Figure 2A) [80]. Another EB1-associated protein, drebrin, has recently emerged as a potential MT-actin crosstalk protein in neurons as this MT-regulating protein promotes the formation of actin bundles in filopodia [81]. Finally, a proteome-wide search revealed MT +TIPs at the actin cortex, such as Growth arrest-specific protein 2 (GAS2) and GAS2 family protein Pigs (binds to actin as well as MTs) [82-84]. In vitro experiments showed that GAS2-like-1 (GAS2L1) exists in an autoinhibited conformation in which the MT binding domain interacts with the actin-binding domain. Upon interaction with MTs, GAS2L1 associates with and stabilizes actin filaments [85]. These results show how MT dynamics at the cell front can control actin polymerization to promote local membrane protrusion and directed cell migration. It also provides insight into the crosstalk of the polarized MT network with actin during migration. 
Whilst MTs influence actin dynamics, the actin network can in turn promote and/or stabilize the organization of MTs, thus creating a feedback loop that maintains cell polarity (Figure 1, 2A). First, actin reorganization facilitates the polarization of the MT network. The balance between the different kinds of stress fibers in a cell, i.e., ventral fibers and transverse arcs, is important for MT organization and polarization [86]. Actin fibers guide MTs to the cell cortex through formins or crosslinking proteins. For example, the actin polymerizing Rho effector proteins of formin family, mDia1 and mDia2, are involved in orienting MTs along the major axis of cells [59, 87]. Inhibition of cell contractility by the depletion of non-muscle myosin IIA or treatment with blebbistatin decreases the levels of mDia1 and mDia2 and promotes MT growth in U2OS osteosarcoma cells [88]. An in vitro study showed that the crosslinking of growing MT plus ends to actin facilitates the alignment and guidance of MTs along pre-existing stiff actin bundles [89]. Along these lines in epithelial cells and keratinocytes, MTs can be guided to the cell front by Actin Crosslinking Factor 7 (ACF7) $[90,91]$, suggesting a close relationship between actin and MTs as they establish their tracks towards the periphery.

In neurons, MT-actin crosstalk is predominantly controlled by Tau, which binds to actin as well as MTs [92]. A recent study demonstrated that Tau not only serves as a MT stabilizing and bundling protein but also interacts with dynamic MTs and promotes their growth along actin bundles towards the actin-rich lamellipodia and filopodia [93]. In addition to Tau, formin 2 (Fmn2) crosslinks MTs with actin, guides MTs along actin bundles and promotes the capture of MTs in filopodia of growth cones [94]. MT capture helps the stabilization of the protrusion and thereby, regulates directionality during neuronal chemotaxis [94]. In neuroblastoma cells, a MTassociated protein (MAP4) induces the elongation of MTs along actin filaments and promotes the formation of protrusions [95].

Once MTs reach the cortex, the actin-rich regions of the cortex act as physical barriers for the interaction of MTs with the membrane (Figure 2B) [83]. Recent in vitro experiments demonstrated that a dense and branched actin meshwork triggers the disassembly of MTs [96], suggesting that the actin-rich cortex in the cell can serve as an obstruction for MT growth, as observed in the actin-rich lamellipodia of migrating cells [97]. In addition to regulating MT growth and polarity, the actomyosin network upon treadmilling induces a rearward movement of MTs, 
excluding them from the leading edge. The buckling of MTs and breaking of MTs due to the actin retrograde flow might also play a role in the polarized organization of the MT network (Figure 2B) $[97,98]$. For example, in neuronal growth cones, the entry of MTs into actin-rich protrusions highly depends upon actin retrograde flow rates and myosin II activity [99]. From these results, it appears that the polarized organization of actin contributes to the guidance and stabilization of MTs from their organizing centers towards the cell front and also influences the dynamics of MTs at the cell periphery.

\subsection{IFs in cytoskeletal crosstalk}

Similar to actin and MTs, perturbations in the IF network can result in the loss of directionality and speed during cell migration [11, 58, 100]; however, the exact link between IFs and polarity is unknown. There is increasing evidence for an interplay between IFs and the actin and MT networks at the protruding cell front. The turnover and polarization of the IF networks involve MTs and MT-associated motors (Figure 2A) [46, 101-103]. Kinesins and dyneins transport IFs along MTs; the preferential binding of kinesins to post-translationally modified acetylated or detyrosinated MTs [104-107] may play a role in the selective transport of IFs to the cell front. Interestingly, oncogene SV40T-induced activity of histone deacetylase 6 (HDAC6; responsible for deacetylating MTs) results in the collapse of the vimentin network [108], confirming the role of MT post-translational modifications in the crosstalk between MTs and IFs. The MT-dependent transport of vimentin is also regulated by actin, ROCK (which inhibits IF transport), Cdc42 and PAK (which stimulate IF transport) [46, 109]. MT-associated protein APC binds to IFs, reorganizes the network, and regulates cell polarity [110]. The direct coupling of IFs and MTs might help template MTs along their previous tracks to promote persistence and polarity [111, 112]. Recent studies identified another cytoskeletal protein Rudhira/Breast Carcinoma Amplified Sequence 3 (BCAS3) as a direct binding partner of MTs and IFs [113-115]. Rudhira controls the crosstalk between MTs and IFs networks to enhance MT stability (acetyl and Glu-tubulin) and cell 
migration [115], possibly through its effects on the master regulator Cdc42 [113]. Thus, similar to actin, the polarized organization of IFs is largely regulated by the MT network (Figure 4).

As vimentin IFs grow towards the cell periphery (Figure 1, 2A), their organization near the cortex was shown to correlate with that of actin $[11,58]$. Actin and IFs are bidirectionally linked; actin retrograde flow affects vimentin subcellular localization, and vimentin depletion results in stronger stress fibers and the disassembly of actin transverse arcs [11, 116-118]. A capping protein, Arp2/3, myosin-I linker 2 (CARMIL2), regulates actin capping protein CP and localizes to vimentin [119]. CARMIL2 was shown to be necessary for polarity, actin assembly and lamellipodium formation, suggesting that vimentin may facilitate actin polymerization at the front through CARMIL2 [119]. These results hint at a contribution of the IFs in the polarization of the actin network (Figure 4) and certainly point towards a need for further investigation in this area.

In conclusion, the mechanisms responsible for the common front-rear orientation of the three cytoskeletal networks in a polarized migrating cell are not only based on common signaling pathways controlling each cytoskeletal network independently (Part 1), but also involve a constant crosstalk between the cytoskeletal structures to ensure their coordination through local regulation and feedback loops (Figure 4).

\section{Cytoskeletal reciprocity in adhesion, contractility and force transmission}

During mesenchymal migration, the dynamics of FAs contribute to the attachment and detachment of cells, and mature FAs transmit forces generated by actomyosin stress fibers onto the substrate [120]. All three cytoskeletal networks influence FA distribution and turnover through a wide range of mechanisms. Briefly, actomyosin contractility promotes FA maturation; MTs facilitate transport to and from FAs to promote FA assembly or disassembly [38, 121]; IFs regulate FA turnover; although whether IFs directly affect FA dynamics and/or influence actin and MTs connecting FAs is not yet entirely clear $[122,123]$. Recent studies have unraveled the mechanisms by which the three cytoskeletal networks, converging at FAs, cooperate to control FA localization, dynamics and force transmission. 


\subsection{Interplay between actin and MTs}

MTs are guided towards the cell front and the dynamic MT plus-ends accumulate at the border between the lamellipodia and lamella where nascent adhesions either disassemble or mature into FAs. In this region, APC promotes actin assembly near FAs, and allows the capture of MTs near FAs (Figure 3A). The APC-mediated capture of MTs in the cortical region results in FA disassembly through autophagosome delivery to FA sites $[124,125]$. Similar to APC, proteins such as ACF7 (which binds actin and MTs to control their dynamics) and Mitogen-activated protein kinase kinase kinase kinase 4 (MAP4K4) might also play a role in the concerted regulation of actin and MTs near stress fiber-linked FAs $[90,126]$.

MTs are stabilized around mature FAs at the cell cortex through the recruitment of the cortical MT stabilizing complex (CMSC) proteins, including the $\mathrm{KN}$ motif and ankyrin repeat domain-containing proteins (KANKs) and the scaffolding proteins liprin- $\alpha 1$ and liprin- $\beta 1$ (Figure $3 A, 4)[36,127]$. KANK proteins interact directly with both talin and liprin- $\beta 1$, thus, linking the CMSC to FAs $[36,128]$. KANK2 activates talin and at the same time, inhibits talin-actomyosin interaction, and thus, reduces force transmission and cell migration [128]. Another study showed that KANK2 decreases the coupling of MTs with $\alpha_{v} \beta_{5}$ containing adhesion complexes, and therefore reduces cell migration [127]. These two studies, although contradictory (possibly due to cell-type specificity), point to KANK proteins as strong links between the actin and MT networks near FAs. The impact of MTs on actomyosin contractility via KANK was also confirmed in a recent study showing that the uncoupling of MTs from adhesions by suppressing KANK expression facilitates the release of MT-bound RhoGEF, Rho guanine nucleotide exchange factor 2 (GEF-H1/ARHGEF2), which activates RhoA and promotes actomyosin contractility (Figure 3A) [22]. The MT-mediated regulation of GEF-H1 and contractility can also occur through Tctex-1, a dynein light chain protein; Tctex-1 recruits GEF-H1 to MTs and therefore acts as a negative regulator of acto-myosin contractility (Figure 3A) [129]. When GEF-H1 is released from MTs, it can activate protein kinase D (PKD), which facilitates Ras-related protein Rab6 delivery to FAs [47]; the Rab6-decorated vesicles dock and fuse with FAs to promote FA turnover [130, 131]. These results demonstrate a role for GEF-H1 in MT-mediated vesicular trafficking and FA 
turnover. It is very likely that the proteins of the CMSC coordinate the stabilization of MTs around FAs and the transmission of forces at these FAs, which would ensure the cooperation of the actin and MT networks at the level of each individual FA.

\subsection{Interplay between IFs and actin}

As cells migrate, IFs polymerize at or extend towards FAs and hemidesmosomes (Figure 3B). In migrating epithelial cells, keratin filament precursors are transported along stress fibers to FAs, where the precursors then form keratin filament networks in the lamellipodia, showing how the interaction of actin with FAs may promote the recruitment of IFs [132].

Uncoupling IFs from FAs either by the loss of vimentin/nestin/Glial fibrillary acidic protein (GFAP) or by depleting the cytoskeletal linker protein, plectin, impairs cell migration [11, 122]. This change in cell migration can be attributed to IFs that alter FA turnover and traction force generation (Figure 3B, 4) $[11,122,133]$. Vimentin- and plectin-depleted cells display increased stress fibers and loss of cell-cell junctions, illustrating the crosstalk between the actin and IF networks [133]. Several mechanisms seem to be involved in this crosstalk. Vimentin which binds to the cytoplasmic tail of $\beta_{3}$ integrin [134], and might directly affect integrin signaling and the connection to actin and MTs. The regulation of FAs by vimentin has also been shown to involve the actin-binding protein filamin A and PKC-mediated phosphorylation of vimentin [135-137]. Moreover, vimentin helps in the recycling and trafficking of integrins to the membrane, thus promoting integrin-ligand binding and FA turnover (Figure 3B) [135, 138]. In addition, IFs can influence the actin network and cell adhesion by manipulating the activity of GEFs involved in the RhoGTPase signaling (Figure 3B). For instance, vimentin induces the phosphorylation of the MTassociated GEF-H1 at Ser886 to trigger Rho activation [139]. Vimentin can also regulate Rac1 activation and the stabilization of focal adhesion kinase (FAK) at FAs through its effects on the activity of RacGEF (VAV2; [140]).

Other IF proteins, nestin and keratins 8 and 18 have also been linked to FA signaling as they recruit FAK to $\beta_{1}$ integrin-mediated FAs $[141,142]$. Desmin IFs are associated with the cortex 
through their interactions with the actin networks surrounding adhesive clathrin plaques. Desmins are recruited to the plaques through clathrin and actin, and desmins can in turn stabilize these clathrin structures [143], which are important for cells to anchor to the substrate and extend protrusions during migration [144].

In epithelial cells, IFs may be able to influence acto-myosin contractility, FAs and cell adhesion through their association with the epithelial-specific $\alpha_{6} \beta_{4}$ containing adhesive structures called hemidesmosomes. In adenocarcinoma cells, the vimentin- $\beta_{4}$ integrin interaction was suggested to reduce Rac1 activity, and therefore, hamper actin polymerization and cell migration [24]. The interaction of keratins with hemidesmosomes reduces FA maturation and traction forces suggesting a crosstalk between IFs and actin in mechanotransduction [145]. Similar to vimentin, evidence indicates that keratins regulate contractility and traction forces during collective cell migration (Figure 3B) [146]. Keratin 6 interacts directly with myosin IIA, regulates FA disassembly, and might also be involved in regulating contractility [147]. Keratins 8 and 18 bind a RhoGEF, Solo, to promote RhoA activation, and thereby the assembly of stress fibers (Figure 3B, 4) [148].

The mechanisms underlying the functions of IFs in controlling FAs and traction forces during migration remain unclear. The complexity involved in the composition of IFs, IF-linked adhesion structures (FAs, adherens junctions, clathrin plaques, hemidesmosomes) and cell-type specificity

triggers unique signaling cascades that could coordinate cytoskeletal functions at FAs and may play a key role in determining the type of migration used by a given cell type. A better characterization of these IF-specific signaling mechanisms will help in understanding the role of IFs in EMT, cancer cell migration, and metastasis.

\section{Concluding remarks and future perspectives}

The individual actions of each cytoskeletal network have been explored well over the last few decades. However, it is the need of the hour to better understand the mechanisms by which they are coordinated during complex processes such as 2D and 3D cell migration. As evidence accumulates supporting the need for precise spatio-temporal cytoskeletal crosstalk during migration (Figure 4), important questions of how the cytoskeletal networks locally interact, either 
through signaling cascades, crosslinking proteins or the mere physical proximity, remain unanswered (see Outstanding Questions). Actin, MTs and IFs extend towards the cell periphery or the leading edge of the cell, helping one another to control and stabilize the cell protrusion and polarization. The signaling pathways are very well characterized in terms of the regulation of the actin cytoskeleton. Actin plays a dominant role in cell contractility; however, the crosstalk between actin, MTs, and IFs in this context appears to be important and needs further investigation (see Outstanding Questions). While the influence of MTs and IFs on actin organization is being unraveled, crucial information on how the actin cytoskeleton conversely influences MTs and IFs still remains unclear.

The identification of downstream targets specific to each cytoskeletal component allows the discrimination of the functions that are unique to the different networks. This will also help decipher how the networks can interact and communicate at a subcellular scale in a spatiotemporal manner (see Outstanding Questions). The interaction of MT +TIPs at the cortex is one example of local crosstalk between actin and MTs at the leading edge of cells. In addition to cytoskeletal linkers or crosslinkers which locally couple cytoskeletal networks, complex signaling cascades involving master cytoskeletal regulators such as the RhoGTPases appear to be involved. These signaling events, precisely regulated by the dynamic behavior of the cytoskeletal elements, are ideal candidates to coordinate subtle readjustments of the cytoskeleton as cells sense their microenvironment and adapt their migratory behavior. Following this hypothesis, the emerging roles of MTs and IFs in controlling FA dynamics and actomyosin contractility suggest that these two cytoskeletal networks may fine-tune mechanotransduction at FAs (see Outstanding Questions). Changes in the composition or the stability of IFs or MTs may influence the ability of cells to migrate on soft or stiff substrates, promote mesenchymal or amoeboid types of migration and play a key role in cell invasion in a physiological context. A deeper understanding of the mechanisms underlying cytoskeletal crosstalk will provide insights on the mechanisms by which alterations in any of the cytoskeletal elements may influence cell migration and in general, cellular behavior during development and in disease. 


\section{Figure legends}

Figure 1. Distribution of the polarized cytoskeletal networks. Mesenchymal cell migration involves five steps - (1) establishment of front-rear polarity, (2) leading edge extension, (3) the formation of new adhesions and their turnover, (4) cell body contraction and (5) rear retraction. The three cytoskeletal networks, actin (in red), microtubules (MTs, in purple) and intermediate filaments (IFs, in cyan-green) polarize in the direction of migration. $(1,2)$ The formation of a protrusion and the corresponding cytoskeletal rearrangements define three major regions of the cell during migration: the cell front/lamellipodium, cell body/lamella and the back of the cell/cell rear. The formation of a lamellipodium and the extension of the leading edge occur through protrusive forces generated by actin polymerization at the cell front. In addition, MTs play a crucial role in the establishment and maintenance of cell polarity, thus defining the directionality during migration. Along with the actin and MT networks, IFs are polarized in the direction of migration. (3) Small nascent adhesions form at the leading edge and mature into actomyosin-linked focal adhesions (FAs) that are seen in the lamella. (4) The association of actin with myosin is crucial for the generation of contractile forces along FA-anchored stress fibers. Actomyosin contractility is mainly governed by the small GTPase Rho and crosstalk mechanisms between the cytoskeletal networks. MT-mediated transport of vesicles to the cell front help in the recycling of membrane proteins as well as signaling molecules, thus contributing vastly to FA turnover and RhoGTPase signaling. In addition to the longitudinal stress fibers, the actin network constantly flows rearward through a process called actin retrograde flow. The IF network appears to protect the cell from the high forces generated during actomyosin contraction. (5) As the cell body contracts, mature FAs start disassembling from the cell rear. The detachment of the cell rear from the substrate promotes rear retraction and the forward movement of the cell.

Figure 2. Schematic depicting cytoskeletal crosstalk during the formation of a lamellipodium and front-rear polarity. A. Cytoskeletal networks extend towards the periphery. Actin, microtubules (MTs) and intermediate filaments (IFs) grow and extend to the cell periphery, and contribute to cell polarization and lamellipodium formation. Actin fibers guide MTs to the cell edge through the formin mDia, actin-microtubule crosslinking protein ACF7, or in a myosin IIdependent manner [89, 90, 149-151]. As MTs grow towards the leading edge, the plus end 
protein EB recruits the CLIP-170-mDia1 complex and promotes MT stabilization and actin polymerization at the front [80]. MT-associated motors transport cargoes containing Cdc42, Rac1 and GEFs to the cell front, thus facilitating signal transduction. Cdc42 and Rac1 at the leading edge help in the establishment and maintenance of polarity as well as actin polymerization. Molecular motors also transport mRNAs of some proteins such as Arp2/3 as well as proteins such as APC and IFs to the cell front, and therefore, indirectly control actin assembly, MT stability and cell polarity $[46,69,72,74-76]$. IFs interact with actin and MTs through the cytoskeletal crosslinking protein plectin, and localize near FAs at the cell front. B. Actin acts as a physical barrier for MT growth. As MTs grow towards the periphery, actin polymerization at the lamellipodium slows down MT growth, causes MT catastrophe or induces MT bending/buckling [152].

Figure 3. Schematic summarizing the cytoskeletal interplay during the regulation of FA turnover and cell contractility. A. Through the crosstalk between MTs and actin. MTs promote actin polymerization through proteins such as APC-stimulated guanine nucleotide exchange factor 1 (Asef), which is a RacGEF associated with APC at the MT plus ends. Local Rac1 activation by Asef at the cell front also promotes FA turnover $[61,153,154]$. The MT-actin crosslinking protein ACF7 is recruited by MAP4K4 to control FA disassembly and FA-actin stability [126]. The FA protein, talin, interacts with KANK $[36,128]$. KANK1 recruits kinesin KIF21A, which inhibits MT growth, and at the same time, prevents catastrophe events to stabilize MTs at the cortex [39]. KANK proteins also inhibit the release of a MT-bound RhoGEF, GEF-H1 into the cytosol, thus, acting as a negative regulator of actomyosin contractility (similar to the effects observed upon nocodazoleinduced MT depolymerization) [22]. When GEF-H1 is released from MTs, it activates RhoA and controls contractility and FA maturation [155-158]. B. Through the crosstalk between IFs and actin/MTs. IFs are transported along MTs and associate with FAs at the leading edge of the cell. IFs alter FA turnover and traction force generation, directly or indirectly through changes in actomyosin contractility $[11,122,133,146]$. Vimentin induces GEF-H1 release from MTs, which triggers Rho activation and actomyosin contractility [139]. Similar to vimentin, keratins can also promote RhoA activation; this occurs through IF-linked RhoGEF Solo [148]. The regulation of FAs by vimentin occurs through actin-binding protein filamin A or PKC-mediated phosphorylation of 
vimentin [135-137]. Phosphorylation (marked as P) of vimentin and MT vesicle transport promote integrin recycling and FA turnover $[135,138]$. Some IF proteins, nestin and keratins 8 and 18 , are also involved in FA signaling as they recruit FAK to FAs [141, 142].

Figure 4. Schematic summarizing the cytoskeletal crosstalk during cell migration. The actomyosin network guides microtubules (MTs) to the cell front in close proximity to focal adhesions (FAs) and stabilizes FA-associated MTs. In turn, MTs regulate contractility and stabilize the cell protrusion. MTs and intermediate filaments (IFs) also share a bidirectional relationship: MTs transport IFs and regulate the assembly and reorganization of the IF network, and IFs stabilize MT orientation. In addition, IFs dampen actomyosin contractility and reduce cell tractions. Reciprocally, the actomyosin network regulates the retrograde flow of IFs. Thus, all three cytoskeletal networks function together to orchestrate cell adhesion and migration.

Figure I (box 2). Mechanisms of 3D migration. This figure depicts the three modes of 3D migration: (A) amoeboid, (B) mesenchymal and (C) lobopodial migration. Cells can switch between the different modes of migration depending on the ECM properties and the changing intracellular and extracellular environments. (A) Amoeboid migration. This involves very few adhesions and low protease activity. Cells migrate through the formation of contraction-based blebs or use actin-driven protrusions to glide on the substrate. The centrosome is usually behind the nucleus during amoeboid migration. (B) Mesenchymal migration. Cells attach very strongly to the ECM through mature stress fiber linked-FAs. These cells also exhibit a high matrix degrading ability. The centrosome is in front of the nucleus, and the three cytoskeletal networks are polarized in the direction of migration. (C) Lobopodial migration. During lobopodial migration, the nucleus acts as a piston that drives migration through the formation of blunt protrusions called lobopodia. These cells exhibit very low protease activity, and high RhoA-ROCKMyoll contractility. 


\section{Box 1: Major functions of actin, microtubules and intermediate filaments in cell migration.}

The dynamics of the cytoskeleton, which comprises actin microfilaments, microtubules (MTs) and intermediate filaments (IFs), play a crucial role in every step of cell migration. In general, cell migration can be divided into five broad steps: establishment of front-rear polarity, leading edge extension, formation of new adhesions, cell body contraction, and rear retraction (Figure 1). Each cytoskeletal network independently contributes to specific properties of one or several migratory steps. First, actin polymerization is generally the major source of protrusive forces allowing the extension of the leading edge, while the association of actin with myosin is crucial for the generation of contractile forces along FA-anchored stress fibers to control the contraction of the cell body and rear retraction. Secondly, MTs are often involved in the establishment and maintenance of cell polarity. MT-dependent transport helps in the delivery of molecules to the leading edge of the cell and to recycle numerous membrane proteins, such as integrins, chemokine receptors or cadherins. These molecules are involved in sensing the microenvironment and controlling FA dynamics and the speed and directionality of migration [127, 159-162]. Thirdly, the role of IFs in cell migration was initially proposed because of their alterations during cancer invasion and progression. Although the exact functions of IFs are not well characterized yet, IFs appear to contribute to the mechanical properties of cells, in order to resist physical constraints and to maintain the integrity of organelles [11, 147, 163, 164].

\section{Box 2: 2D and 3D cell migration}

Cell migration in 2D has been extensively studied over the last few decades. However, cells in the body experience a complex 3D microenvironment and they have to adapt accordingly in order to perform different cell type-dependent functions. Similarities and differences between cell migration in 2D and in 3D have been found. Figure I depicts the three modes of 3D migration: amoeboid (Figure IA), mesenchymal (Figure IB) and lobopodial migration (Figure IC).

In contrast to 2D migration, 3D migration largely depends on the properties of the surrounding environment, such as chemical cues, ligand specificity, rigidity of the matrix, pore size that cells have to navigate through, as well as the topology of the microenvironment. Cells sense the properties of the environment through integrins [165-167], extend protrusions such as 
lobopodia, invadopodia and filopodia [16, 168-171], and explore different regions in the surrounding ECM before adopting a migration strategy (Figure I). Cells in 3D experience confinement and they migrate by degrading proteins in the ECM and remodeling the matrix (distinguished by protease activity on Figure I). RhoA-ROCK-mediated actomyosin contractility controls cell adhesion and the different modes of migration through the 3D matrix (Figure I) [10, 172-174]. Similar to 2D, integrin-mediated FAs are observed during 3D migration (Figure IB) [169]. In addition, the nucleus being highly bulky poses challenges when cells squeeze through the matrix and this can cause significant DNA damage [175-178]. However, in some cases, the lack of a nucleus also slows down migration [179].

Another characteristic of 3D cell migration involves the rapid switch between the different modes of migration. Upon confinement and high actomyosin contractility, cells can switch from mesenchymal to amoeboid modes of migration using less adhesions (Figure IA, B) [14, 15]. Cells can also exhibit plasticity as they switch from amoeboid or lobopodial to mesenchymal type of migration $[16,180]$.

Similar to 2D systems, the centrosome is often positioned in front of the nucleus in 3D mesenchymal migration; however, the centrosome is frequently observed behind the nucleus in 3D amoeboid migration [64, 181, 182]. Gradients of intracellular proteins such as Cdc42, Rac1 and Phosphatidylinositol $(3,4,5)$-trisphosphate (PtdIns(3,4,5)P3 or PIP3) play similar roles in 3D mesenchymal migration as in 2D; however, in some modes of 3D migration such as the nuclear piston driven migration (Figure IC), these signaling molecules are observed in a non-polarized manner [183].

\section{Acknowledgements}

This work was supported by the La Ligue contre le cancer (S-CR17017), Centre National de la Recherche Scientifique and Institut Pasteur. SS is funded by the ITN PolarNet Marie Curie grant and Fondation pour la Recherche Médicale and is enrolled at the Ecole Doctorale Frontières du 
Vivant (FdV) - Programme Bettencourt. We would like to thank Emma van Bodegraven for her critical reading of the manuscript and discussions.

\section{Glossary}

+TIP proteins: MT plus-end tracking proteins such as EB1.

Actin: Cytoskeletal protein existing as a monomer globular actin (G-actin) in equilibrium with a helicoidal polymer of filamentous actin (F-actin). Actin associates with myosin II motors to form the actomyosin network, which generates contractile forces.

Cortical microtubule stabilizing complex (CMSC): complex of FA-associated proteins involved in stabilizing microtubules at the cell cortex. A few proteins of this complex are KANKs, LL5 $\beta$ and liprins.

Extracellular matrix (ECM): mix of fibrous proteins, viscous proteoglycans and polysaccharides, which are secreted by cells and surround them.

Focal adhesions (FAs): adhesive structures based on core transmembrane proteins of the integrin family. They also include multiple cytoplasmic proteins such as talin, vinculin and paxillin, which link the integrins to the actomyosin cytoskeleton. FAs contribute to the attachment of cells to the substrate/ECM.

Integrins: heterodimeric trans-membrane receptors that interact with fibrous proteins of the ECM. Integrin engagement with the ECM triggers downstream signaling cascades that promote the formation of FAs and regulate cell adhesion and migration.

Intermediate filaments (IFs): dense filamentous cytoskeletal network present in most animal cells. Depending on the cell type and differentiation state, IFs are composed of one or several of the more than 70 IF proteins, including keratins and vimentin. 
Lamellipodium: Dynamic membrane protrusion, rich in branched actin.

Mechanotransduction: multi-step process by which the mechanical properties of the environment are sensed by molecular mechanosensors (mechanosensing) and transduced into biochemical intracellular signals which impact the cytoskeleton and signaling cascades.

Microtubule-associated motors: proteins (kinesins and dyneins) which use ATP to "walk" in a directed manner along microtubules. They are responsible for the transport of various types of cargoes around the cell.

Microtubules (MTs): hollowed tubes formed of protofilaments (13 in general) of tubulin dimers. These highly dynamic cytoskeletal structures undergo continuous assembly and disassembly.

Stress fibers: contractile actomyosin fibers linked to FAs.

\section{References}

1 Mayor, R. and Etienne-Manneville, S. (2016) The front and rear of collective cell migration. Nat. Rev. Mol. Cell Biol. 17, 97-109

2 Barzilai, S., et al. (2017) Leukocytes breach endothelial barriers by insertion of nuclear lobes and disassembly of endothelial actin filaments. Cell Rep. 18, 685-699

3 Prentice-Mott, H.V., et al. (2016) Directional memory arises from long-lived cytoskeletal asymmetries in polarized chemotactic cells. Proc. Natl. Acad. Sci. U. S. A. 113, 1267-1272

4 Van Helvert, S., et al. (2018) Mechanoreciprocity in cell migration. Nat. Cell Biol. 20, 8-20

5 Davidson, A.J., et al. (2017) WASP family proteins and formins compete in pseudopod-and bleb-based migration. J. Cell Biol. 217, 701-714

6 Gong, X., et al. (2018) KIF13A-regulated RhoB plasma membrane localization governs membrane blebbing and blebby amoeboid cell migration. EMBO J. 37, e98994

7 Lavenus, S.B., et al. (2020) A flexible network of Vimentin intermediate filaments promotes the migration of amoeboid cancer cells through confined environments. J. Biol. Chem. 295, 6700-6709

8 O'Neill, P.R., et al. (2018) Membrane Flow Drives an Adhesion-Independent Amoeboid Cell Migration Mode. Dev. Cell 46, 9-22.e24

9 Renkawitz, J., et al. (2019) Nuclear positioning facilitates amoeboid migration along the path of least resistance. Nature 568, 546-550

10 Wang, W.Y., et al. (2019) Actomyosin contractility-dependent matrix stretch and recoil induces rapid cell migration. Nat. Commun. 10, 1-12 
11 De Pascalis, C., et al. (2018) Intermediate filaments control collective migration by restricting traction forces and sustaining cell-cell contacts. J. Cell Biol. 217, 3031-3044

12 Ray, A., et al. (2017) Anisotropic forces from spatially constrained focal adhesions mediate contact guidance directed cell migration. Nat. Commun. 8, 14923

13 Fokkelman, M., et al. (2016) Cellular adhesome screen identifies critical modulators of focal adhesion dynamics, cellular traction forces and cell migration behaviour. Sci. Rep. 6, 31707

14 Liu, Y.-J., et al. (2015) Confinement and low adhesion induce fast amoeboid migration of slow mesenchymal cells. Cell 160, 659-672

15 Ruprecht, V., et al. (2015) Cortical contractility triggers a stochastic switch to fast amoeboid cell motility. Cell 160, 673-685

16 Petrie, R.J., et al. (2017) Activating the nuclear piston mechanism of 3D migration in tumor cells. J. Cell Biol. 216, 93-100

17 Bouchet, B.P. and Akhmanova, A. (2017) Microtubules in 3D cell motility. J. Cell Sci. 130, 39-50

18 Etienne-Manneville, S. (2018) Cytoplasmic Intermediate Filaments in Cell Biology. Annu. Rev. Cell. Dev. Biol. 34, 1-28

19 Schaks, M., et al. (2019) Actin dynamics in cell migration. Essays Biochem. 63, 483-495

20 Lawson, C.D. and Ridley, A.J. (2018) Rho GTPase signaling complexes in cell migration and invasion. J. Cell Biol. 217, 447-457

21 Cheng, H.-W., et al. (2019) Centrosome guides spatial activation of Rac to control cell polarization and directed cell migration. Life Sci. Alliance 2, e201800135

22 Rafiq, N.B.M., et al. (2019) A mechano-signalling network linking microtubules, myosin IIA filaments and integrin-based adhesions. Nat. Mater. 18, 638-649

23 Zinn, A., et al. (2019) The small GTPase RhoG regulates microtubule-mediated focal adhesion disassembly. Sci. Rep. 9, 5163

24 Colburn, Z.T. and Jones, J.C.R. (2018) Complexes of alpha6beta4 integrin and vimentin act as signaling hubs to regulate epithelial cell migration. J. Cell Sci. 131, jcs214593

25 Nobes, C.D., et al. (1998) A new member of the Rho family, Rnd1, promotes disassembly of actin filament structures and loss of cell adhesion. J. Cell Biol. 141, 187-197

26 Riento, K., et al. (2003) RhoE Binds to ROCK I and Inhibits Downstream Signaling. Mol. Cell. Biol. 23, 4219-4229

27 Tajadura-Ortega, V., et al. (2018) An RNAi screen of Rho signalling networks identifies RhoH as a regulator of Rac1 in prostate cancer cell migration. BMC Biol. 16, 29

28 Dart, A.E., et al. (2015) PAK4 promotes kinase-independent stabilization of RhoU to modulate cell adhesion. J. Cell Biol. 211, 863-879

29 Blom, M., et al. (2017) The atypical Rho GTPase RhoD is a regulator of actin cytoskeleton dynamics and directed cell migration. Exp. Cell Res. 352, 255-264

$30 \mathrm{Gad}$, A.K., et al. (2012) RhoD regulates cytoskeletal dynamics via the actin nucleation-promoting factor WASp homologue associated with actin Golgi membranes and microtubules. Mol. Biol. Cell 23, 4807-4819 31 Machacek, M., et al. (2009) Coordination of Rho GTPase activities during cell protrusion. Nature 461, 99-103

32 Martin, K., et al. (2016) Spatio-temporal co-ordination of RhoA, Rac1 and Cdc42 activation during prototypical edge protrusion and retraction dynamics. Sci. Rep. 6, 1-14

33 Müller, P.M., et al. (2020) Systems analysis of RhoGEF and RhoGAP regulatory proteins reveals spatially organized RAC1 signalling from integrin adhesions. Nat. Cell Biol. 22, 498-511

34 O'Neill, P.R., et al. (2016) Subcellular optogenetic activation of Cdc42 controls local and distal signaling to drive immune cell migration. Mol. Biol. Cell 27, 1442-1450

35 Yang, H.W., et al. (2016) Locally excitable Cdc42 signals steer cells during chemotaxis. Nat. Cell Biol. 18, 191-201 
36 Bouchet, B.P., et al. (2016) Talin-KANK1 interaction controls the recruitment of cortical microtubule stabilizing complexes to focal adhesions. Elife 5, e18124

37 Lovelace, M.D., et al. (2017) The RhoGAP protein ARHGAP18/SENEX localizes to microtubules and regulates their stability in endothelial cells. Mol. Biol. Cell 28, 1066-1078

38 Seetharaman, S. and Etienne-Manneville, S. (2019) Microtubules at focal adhesions - a double-edged sword. J. Cell Sci. 132, jcs232843

39 van der Vaart, B., et al. (2013) CFEOM1-Associated Kinesin KIF21A Is a Cortical Microtubule Growth Inhibitor. Dev. Cell 27, 145-160

40 Vestre, K., et al. (2019) Rab6 regulates cell migration and invasion by recruiting Cdc42 and modulating its activity. Cell. Mol. Life Sci. 76, 2593-2614

41 Etienne-Manneville, S., et al. (2005) Cdc42 and Par6-PKC regulate the spatially localized association of DIg1 and APC to control cell polarization. J. Cell Biol. 170, 895-901

42 Baschieri, F., et al. (2014) Spatial control of Cdc42 signalling by a GM130-RasGRF complex regulates polarity and tumorigenesis. Nat. Commun. 5, 1-12

43 Bhattacharya, K., et al. (2016) Novel Coronin7 interactions with Cdc42 and N-WASP regulate actin organization and Golgi morphology. Sci. Rep. 6, 25411

44 Farhan, H. and Hsu, V.W. (2016) Cdc42 and Cellular Polarity: Emerging Roles at the Golgi. Trends Cell Biol. 26, 241-248

45 Urrutia, P.J., et al. (2019) Tuba activates Cdc42 during neuronal polarization downstream of the small GTPase Rab8a. bioRxiv, 2019.2012.2016.876011

46 Leduc, C. and Etienne-Manneville, S. (2017) Regulation of microtubule-associated motors drives intermediate filament network polarization. J. Cell Biol. 216, 1689-1703

47 Eisler, S.A., et al. (2018) A Rho signaling network links microtubules to PKD controlled carrier transport to focal adhesions. eLife 7, p.e35907

48 Fukata, M., et al. (2002) Rac1 and Cdc42 capture microtubules through IQGAP1 and CLIP-170. Cell 109, 873-885

49 Rooney, C., et al. (2010) The Rac activator STEF (Tiam2) regulates cell migration by microtubulemediated focal adhesion disassembly. EMBO Rep. 11, 292-298

50 Wittmann, T., et al. (2003) Regulation of leading edge microtubule and actin dynamics downstream of Rac1. J. Cell Biol. 161, 845-851

51 Amato, C., et al. (2019) WASP Restricts Active Rac to Maintain Cells' Front-Rear Polarization. Curr. Biol. 29, 4169-4182.e4164

52 Fort, L., et al. (2018) Fam49/CYRI interacts with Rac1 and locally suppresses protrusions. Nat. Cell Biol. 20, 1159-1171

53 Daub, H., et al. (2001) Rac/Cdc42 and p65PAK regulate the microtubule-destabilizing protein stathmin through phosphorylation at serine 16. J. Biol. Chem. 276, 1677-1680

54 Wittmann, T., et al. (2004) Regulation of microtubule destabilizing activity of Op18/stathmin downstream of Rac1. J. Biol. Chem. 279, 6196-6203

55 Helfand, B.T., et al. (2011) Vimentin organization modulates the formation of lamellipodia. Mol. Biol. Cell 22, 1274-1289

56 Goto, H., et al. (1998) Phosphorylation of Vimentin by Rho-associated Kinase at a Unique Aminoterminal Site That Is Specifically Phosphorylated during Cytokinesis. J. Biol. Chem. 273, 11728-11736

57 Hyder, C.L., et al. (2015) Sphingolipids inhibit vimentin-dependent cell migration. J. Cell Sci. 128, 20572069

58 Terriac, E., et al. (2017) Vimentin Levels and Serine 71 Phosphorylation in the Control of Cell-Matrix Adhesions, Migration Speed, and Shape of Transformed Human Fibroblasts. Cells 6, 2

59 Palazzo, A.F., et al. (2001) mDia mediates Rho-regulated formation and orientation of stable microtubules. Nat. Cell Biol. 3, 723-729 
60 Palazzo, A.F., et al. (2004) Localized stabilization of microtubules by integrin- and FAK-facilitated Rho signaling. Science 303, 836-839

61 Wen, Y., et al. (2004) EB1 and APC bind to mDia to stabilize microtubules downstream of Rho and promote cell migration. Nat. Cell Biol. 6, 820-830

62 Sudarsanam, S., et al. (2020) Cofilin regulates axon growth and branching of Drosophila y neurons. J. Cell Sci., jcs.232595

$63 \mathrm{Li}, \mathrm{X}$., et al. (2020) Excitable networks controlling cell migration during development and disease. Semin. Cell Dev. Biol. 100, 133-142

64 Meiring, J.C.M., et al. (2019) Generation and regulation of microtubule network asymmetry to drive cell polarity. Curr. Opin. Cell Biol. 62, 86-95

65 Nishita, M., et al. (2017) Regulatory mechanisms and cellular functions of non-centrosomal microtubules. J. Biochem. 162, 1-10

66 Burute, M., et al. (2017) Polarity Reversal by Centrosome Repositioning Primes Cell Scattering during Epithelial-to-Mesenchymal Transition. Dev. Cell 40, 168-184

67 Jimenez, A.J., et al. (2020) Acto-myosin network geometry defines centrosome position. bioRxiv, 2020.2001.2007.896969

68 Martin, M., et al. (2018) Control of endothelial cell polarity and sprouting angiogenesis by noncentrosomal microtubules. Elife 7, e33864

69 Mardakheh, F.K., et al. (2015) Global analysis of mRNA, translation, and protein localization: local translation is a key regulator of cell protrusions. Dev. Cell 35, 344-357

70 Mili, S., et al. (2008) Genome-wide screen reveals APC-associated RNAs enriched in cell protrusions. Nature 453, 115-119

71 Mingle, L.A., et al. (2005) Localization of all seven messenger RNAs for the actin-polymerization nucleator Arp2/3 complex in the protrusions of fibroblasts. J. Cell Sci. 118, 2425-2433

72 Ruane, P.T., et al. (2016) Tumour Suppressor Adenomatous Polyposis Coli (APC) localisation is regulated by both Kinesin-1 and Kinesin-2. Sci. Rep. 6, 27456

73 Liao, G., et al. (2011) Mis-localization of Arp2 mRNA impairs persistence of directional cell migration. Exp. Cell Res. 317, 812-822

74 Wang, T., et al. (2017) Extracellular matrix stiffness and cell contractility control RNA localization to promote cell migration. Nat. Commun. 8, 1-16

75 Osmani, N., et al. (2010) Cdc42 localization and cell polarity depend on membrane traffic. J. Cell Biol. $191,1261-1269$

76 Palamidessi, A., et al. (2008) Endocytic trafficking of Rac is required for the spatial restriction of signaling in cell migration. Cell 134, 135-147

77 Waterman-Storer, C.M., et al. (1999) Microtubule growth activates Rac1 to promote lamellipodial protrusion in fibroblasts. Nat. Cell Biol. 1, 45-50

78 Bershadsky, A., et al. (1996) Involvement of microtubules in the control of adhesion-dependent signal transduction. Curr. Biol. 6, 1279-1289

79 Campellone, K.G., et al. (2008) WHAMM is an Arp2/3 complex activator that binds microtubules and functions in ER to Golgi transport. Cell 134, 148-161

80 Henty-Ridilla, J.L., et al. (2016) Accelerated actin filament polymerization from microtubule plus ends. Science 352, 1004-1009

81 Ketschek, A., et al. (2016) Drebrin coordinates the actin and microtubule cytoskeleton during the initiation of axon collateral branches. Dev. Neurobiol. 76, 1092-1110

82 Stroud, M.J., et al. (2014) GAS2-like proteins mediate communication between microtubules and actin through interactions with end-binding proteins. J. Cell Sci. 127, 2672-2682

83 Jiang, K., et al. (2012) A Proteome-wide Screen for Mammalian SxIP Motif-Containing Microtubule PlusEnd Tracking Proteins. Curr. Biol. 22, 1800-1807 
84 Girdler, G.C., et al. (2016) The Gas2 family protein Pigs is a microtubule+ TIP that affects cytoskeleton organisation. J. Cell Sci. 129, 121-134

85 van de Willige, D., et al. (2019) Cytolinker Gas2L1 regulates axon morphology through microtubulemodulated actin stabilization. EMBO Rep. 20, e47732

86 Tabdanov, E.D., et al. (2018) Microtubule-Actomyosin Mechanical Cooperation during Contact Guidance Sensing. Cell Rep. 25, 328-338.e325

87 Ishizaki, T., et al. (2001) Coordination of microtubules and the actin cytoskeleton by the Rho effector mDia1. Nat. Cell Biol. 3, 8-14

88 Sato, Y., et al. (2020) Nonmuscle myosin IIA and IIB differently suppress microtubule growth to stabilize cell morphology. J. Biochem. 167, 25-39

89 López, M.P., et al. (2014) Actin-microtubule coordination at growing microtubule ends. Nat. Commun. $5,1-9$

90 Ning, W., et al. (2016) The CAMSAP3-ACF7 Complex Couples Noncentrosomal Microtubules with Actin Filaments to Coordinate Their Dynamics. Dev. Cell 39, 61-74

$91 \mathrm{Wu}, \mathrm{X}$., et al. (2008) ACF7 Regulates Cytoskeletal-Focal Adhesion Dynamics and Migration and Has ATPase Activity. Cell 135, 137-148

92 Elie, A., et al. (2015) Tau co-organizes dynamic microtubule and actin networks. Sci. Rep. 5, 9964

93 Biswas, S. and Kalil, K. (2018) The microtubule-associated protein tau mediates the organization of microtubules and their dynamic exploration of actin-rich lamellipodia and filopodia of cortical growth cones. J. Neurosci. 38, 291-307

94 Kundu, T., et al. (2020) Coupling of dynamic microtubules to F-actin by Fmn2 regulates chemotaxis of neuronal growth cones. bioRxiv, 2020.2001.2018.911131

95 Doki, C., et al. (2020) Microtubule elongation along actin filaments induced by microtubule-associated protein 4 contributes to the formation of cellular protrusions. J. Biochem., mvaa046

96 Colin, A., et al. (2018) Actin-Network Architecture Regulates Microtubule Dynamics. Curr. Biol. 28, 2647-2656.e2644

97 Waterman-Storer, C.M. and Salmon, E. (1997) Actomyosin-based retrograde flow of microtubules in the lamella of migrating epithelial cells influences microtubule dynamic instability and turnover and is associated with microtubule breakage and treadmilling. J. Cell Biol. 139, 417-434

98 Even-Ram, S., et al. (2007) Myosin IIA regulates cell motility and actomyosin-microtubule crosstalk. Nat. Cell Biol. 9, 299-309

99 Turney, S.G., et al. (2020) Variation and selection in axon navigation through microtubule-dependent stepwise growth cone advance. bioRxiv, 2020.2001.2029.925602

100 Moeton, M., et al. (2014) Silencing GFAP isoforms in astrocytoma cells disturbs laminin-dependent motility and cell adhesion. FASEB J. 28, 2942-2954

101 Gyoeva, F.K. and Gelfand, V.I. (1991) Coalignment of vimentin intermediate filaments with microtubules depends on kinesin. Nature 353, 445-448

102 Helfand, B.T., et al. (2002) A requirement for cytoplasmic dynein and dynactin in intermediate filament network assembly and organization. J. Cell Biol. 157, 795-806

103 Hookway, C., et al. (2015) Microtubule-dependent transport and dynamics of vimentin intermediate filaments. Mol. Biol. Cell 26, 1675-1686

104 Balabanian, L., et al. (2017) Acetylated Microtubules Are Preferentially Bundled Leading to Enhanced Kinesin-1 Motility. Biophys. J. 113, 1551-1560

105 Liao, G. and Gundersen, G.G. (1998) Kinesin is a candidate for cross-bridging microtubules and intermediate filaments selective binding of Kinesin to detyrosinated tubulin and vimentin. J. Biol. Chem. 273, 9797-9803

106 Reed, N.A., et al. (2006) Microtubule Acetylation Promotes Kinesin-1 Binding and Transport. Curr. Biol. 16, 2166-2172 
107 Tas, R.P., et al. (2017) Differentiation between Oppositely Oriented Microtubules Controls Polarized Neuronal Transport. Neuron 96, 1264-1271.e1265

108 Rathje, L.S., et al. (2014) Oncogenes induce a vimentin filament collapse mediated by HDAC6 that is linked to cell stiffness. Proc. Natl. Acad. Sci. U. S. A. 111, 1515-1520

109 Robert, A., et al. (2014) Microtubule-dependent transport of vimentin filament precursors is regulated by actin and by the concerted action of Rho- and p21-activated kinases. FASEB J. 28, 2879-2890 110 Sakamoto, Y., et al. (2013) APC binds intermediate filaments and is required for their reorganization during cell migration. J. Cell Biol. 200, 249-258

111 Gan, Z., et al. (2016) Vimentin Intermediate Filaments Template Microtubule Networks to Enhance Persistence in Cell Polarity and Directed Migration. Cell systems 3, 252-263.e258

112 Shabbir, S.H., et al. (2014) Geometric control of vimentin intermediate filaments. Biomaterials 35, 1359-1366

113 Jain, M., et al. (2012) Rudhira/BCAS3 is a cytoskeletal protein that controls Cdc42 activation and directional cell migration during angiogenesis. Exp. Cell Res. 318, 753-767

114 Shetty, R., et al. (2018) Rudhira/BCAS3 is essential for mouse development and cardiovascular patterning. Sci. Rep. 8, 1-12

115 Joshi, D. and Inamdar, M.S. (2019) Rudhira/BCAS3 couples microtubules and intermediate filaments to promote cell migration for angiogenic remodeling. Mol. Biol. Cell 30, 1437-1450

$116 \mathrm{Jiu}, \mathrm{Y}$., et al. (2015) Bidirectional interplay between vimentin intermediate filaments and contractile actin stress fibers. Cell Rep. 11, 1511-1518

117 Bhattacharya, R., et al. (2009) Recruitment of vimentin to the cell surface by $\beta 3$ integrin and plectin mediates adhesion strength. J. Cell Sci. 122, 1390-1400

118 Eckes, B., et al. (1998) Impaired mechanical stability, migration and contractile capacity in vimentindeficient fibroblasts. J. Cell Sci. 111, 1897-1907

119 Lanier, M.H., et al. (2015) CARMIL2 is a novel molecular connection between vimentin and actin essential for cell migration and invadopodia formation. Mol. Biol. Cell 26, 4577-4588

120 Conway, J.R.W. and Jacquemet, G. (2019) Cell matrix adhesion in cell migration. Essays Biochem. 63, 535-551

121 Fourriere, L., et al. (2019) RAB6 and microtubules restrict protein secretion to focal adhesions. J. Cell Biol. 218, 2215-2231

122 Gregor, M., et al. (2014) Mechanosensing through focal adhesion-anchored intermediate filaments. FASEB J. 28, 715-729

123 Sanghvi-Shah, R. and Weber, G.F. (2017) Intermediate Filaments at the Junction of Mechanotransduction, Migration, and Development. Front. Cell Dev. Biol. 5, 81

124 Juanes, M.A. and Bouguenina, H. (2017) Adenomatous polyposis coli nucleates actin assembly to drive cell migration and microtubule-induced focal adhesion turnover. J. Cell Biol. 216, 2859-2875

125 Juanes, M.A., et al. (2019) The role of APC-mediated actin assembly in microtubule capture and focal adhesion turnover. Journal of Cell Biology 218, 3415-3435

126 Yue, J., et al. (2014) Microtubules regulate focal adhesion dynamics through MAP4K4. Dev. Cell 31, 572-585

127 Paradžik, M., et al. (2020) KANK2 Links aVß5 Focal Adhesions to Microtubules and Regulates Sensitivity to Microtubule Poisons and Cell Migration. Front. Cell Dev. Biol. 8,125

128 Sun, Z., et al. (2016) Kank2 activates talin, reduces force transduction across integrins and induces central adhesion formation. Nat. Cell Biol. 18, 941-953

129 Meiri, D., et al. (2012) Mechanistic Insight into the Microtubule and Actin Cytoskeleton Coupling through Dynein-Dependent RhoGEF Inhibition. Mol. Cell 45, 642-655

130 Grigoriev, I., et al. (2011) Rab6, Rab8, and MICAL3 cooperate in controlling docking and fusion of exocytotic carriers. Curr. Biol. 21, 967-974 
131 Stehbens, S.J., et al. (2014) CLASPs link focal-adhesion-associated microtubule capture to localized exocytosis and adhesion site turnover. Nat. Cell Biol. 16, 558-570

132 Windoffer, R., et al. (2006) Focal adhesions are hotspots for keratin filament precursor formation. J. Cell Biol. 173, 341-348

133 Osmanagic-Myers, S., et al. (2015) Plectin reinforces vascular integrity by mediating crosstalk between the vimentin and the actin networks. J. Cell Sci. 128, 4138-4150

$134 \mathrm{Kim}, \mathrm{J} .$, et al. (2016) Vimentin filaments regulate integrin-ligand interactions by binding to the cytoplasmic tail of integrin beta3. J. Cell Sci. 129, 2030-2042

135 Ivaska, J., et al. (2005) PKCE-mediated phosphorylation of vimentin controls integrin recycling and motility. EMBO J. 24, 3834-3845

$136 \mathrm{Kim}, \mathrm{H}$., et al. (2010) Filamin A is required for vimentin-mediated cell adhesion and spreading. Am. J. Physiol. Cell Physiol. 298, C221-C236

$137 \mathrm{Kim}, \mathrm{H}$., et al. (2010) Regulation of cell adhesion to collagen via $\beta 1$ integrins is dependent on interactions of filamin A with vimentin and protein kinase C epsilon. Exp. Cell Res. 316, 1829-1844

138 Ivaska, J., et al. (2007) Novel functions of vimentin in cell adhesion, migration, and signaling. Exp. Cell Res. 313, 2050-2062

$139 \mathrm{Jiu}, \mathrm{Y}$., et al. (2017) Vimentin intermediate filaments control actin stress fiber assembly through GEF$\mathrm{H} 1$ and RhoA. J Cell Sci 130, 892-902

140 Havel, L.S., et al. (2015) Vimentin regulates lung cancer cell adhesion through a VAV2-Rac1 pathway to control focal adhesion kinase activity. Oncogene 34, 1979-1990

141 Bordeleau, F., et al. (2010) Keratin 8/18 Modulation of Protein Kinase C-mediated Integrin-dependent Adhesion and Migration of Liver Epithelial Cells. Mol. Biol. Cell 21, 1698-1713

142 Hyder, C.L., et al. (2014) Nestin regulates prostate cancer cell invasion by influencing the localisation and functions of FAK and integrins. J. Cell Sci. 127, 2161-2173

143 Franck, A., et al. (2019) Clathrin plaques and associated actin anchor intermediate filaments in skeletal muscle. Mol. Biol. Cell 30, 579-590

144 Elkhatib, N., et al. (2017) Tubular clathrin/AP-2 lattices pinch collagen fibers to support 3D cell migration. Science 356, eaal4713

145 Wang, W., et al. (2020) Hemidesmosomes modulate force generation via focal adhesions. J. Cell Biol. 219(2). pii: e201904137.

146 Sonavane, P.R., et al. (2017) Mechanical and signaling roles for keratin intermediate filaments in the assembly and morphogenesis of Xenopus mesendoderm tissue at gastrulation. Development 144, 43634376

147 Wang, F., et al. (2018) Keratin 6 regulates collective keratinocyte migration by altering cell-cell and cell-matrix adhesion. J. Cell Biol. 217, 4314-4330

148 Fujiwara, S., et al. (2016) Interplay between Solo and keratin filaments is crucial for mechanical forceinduced stress fiber reinforcement. Mol. Biol. Cell 27, 954-966

149 Margaron, Y., et al. (2013) ELMO recruits actin cross-linking family 7 (ACF7) at the cell membrane for microtubule capture and stabilization of cellular protrusions. J. Biol. Chem. 288, 1184-1199

$150 \mathrm{Wu}, \mathrm{X}$., et al. (2011) Skin stem cells orchestrate directional migration by regulating microtubule-ACF7 connections through GSK3beta. Cell 144, 341-352

151 Huda, S., et al. (2012) Microtubule guidance tested through controlled cell geometry. J. Cell Sci. 125, 5790-5799

152 Dogterom, M. and Koenderink, G.H. (2019) Actin-microtubule crosstalk in cell biology. Nat. Rev. Mol. Cell Biol. 20, 38-54

153 Breitsprecher, D., et al. (2012) Rocket Launcher Mechanism of Collaborative Actin Assembly Defined by Single-Molecule Imaging. Science 336, 1164-1168 
154 Mimori-Kiyosue, Y., et al. (2000) Adenomatous polyposis coli (APC) protein moves along microtubules and concentrates at their growing ends in epithelial cells. J. Cell Biol. 148, 505-518

155 Chang, Y.C., et al. (2008) GEF-H1 couples nocodazole-induced microtubule disassembly to cell contractility via RhoA. Mol. Biol. Cell 19, 2147-2153

156 Guilluy, C., et al. (2011) The Rho GEFs LARG and GEF-H1 regulate the mechanical response to force on integrins. Nat. Cell Biol. 13, 722-727

157 Krendel, M., et al. (2002) Nucleotide exchange factor GEF-H1 mediates cross-talk between microtubules and the actin cytoskeleton. Nat. Cell Biol. 4, 294-301

158 Yoshimura, Y. and Miki, H. (2011) Dynamic regulation of GEF-H1 localization at microtubules by Par1b/MARK2. Biochem. Biophys. Res. Commun. 408, 322-328

159 Belvindrah, R., et al. (2017) Mutation of the $\alpha$-tubulin Tuba1a leads to straighter microtubules and perturbs neuronal migration. J. Cell Biol. 216, 2443-2461

$160 \mathrm{HaO}, \mathrm{H}$. , et al. (2020) Golgi-associated microtubules are fast cargo tracks and required for persistent cell migration. EMBO Rep. 21, e48385

$161 \mathrm{Hu}, \mathrm{Z}$., et al. (2017) Fidgetin regulates cultured astrocyte migration by severing tyrosinated microtubules at the leading edge. Mol. Biol. Cell 28, 545-553

162 Bance, B., et al. (2019) Microtubule acetylation but not detyrosination promotes focal adhesion dynamics and astrocyte migration. J. Cell Sci. 132, jcs.225805

163 Holle, A.W., et al. (2017) Intermediate filament reorganization dynamically influences cancer cell alignment and migration. Sci. Rep. 7, 45152

164 Block, J., et al. (2015) Physical properties of cytoplasmic intermediate filaments. Biochim. Biophys. Acta 1853, 3053-3064

165 Mekhdjian, A.H., et al. (2017) Integrin-mediated traction force enhances paxillin molecular associations and adhesion dynamics that increase the invasiveness of tumor cells into a three-dimensional extracellular matrix. Mol. Biol. Cell 28, 1467-1488

166 Kechagia, J.Z., et al. (2019) Integrins as biomechanical sensors of the microenvironment. Nat. Rev. Mol. Cell Biol. 20(8), 457-473

167 Wisdom, K.M., et al. (2018) Matrix mechanical plasticity regulates cancer cell migration through confining microenvironments. Nat. Commun. 9, 1-13

168 Erdogan, B., et al. (2017) Cancer-associated fibroblasts promote directional cancer cell migration by aligning fibronectin. J. Cell Biol. 216, 3799-3816

169 Doyle, A.D., et al. (2015) Local 3D matrix microenvironment regulates cell migration through spatiotemporal dynamics of contractility-dependent adhesions. Nat. Commun. 6, 1-15

170 lizuka, S., et al. (2020) Crosstalk between invadopodia and the extracellular matrix. bioRxiv, 2020.2002.2026.966762

171 Franchi, M., et al. (2020) Long filopodia and tunneling nanotubes define new phenotypes of breast cancer cells in 3D cultures. Matrix Biol., 100026

172 Carmona, G., et al. (2016) Lamellipodin promotes invasive 3D cancer cell migration via regulated interactions with Ena/VASP and SCAR/WAVE. Oncogene 35, 5155

173 Pfisterer, K., et al. (2020) FMNL2 regulates dynamics of fascin in filopodia. J. Cell Biol. 219

174 Sao, K., et al. (2019) Myosin II governs intracellular pressure and traction by distinct tropomyosindependent mechanisms. Mol. Biol. Cell 30, 1170-1181

175 Irianto, J., et al. (2017) DNA damage follows repair factor depletion and portends genome variation in cancer cells after pore migration. Curr. Biol. 27, 210-223

176 Zhang, Q., et al. (2019) Local, transient tensile stress on the nuclear membrane causes membrane rupture. Mol. Biol. Cell 30, 899-906

177 Denais, C.M., et al. (2016) Nuclear envelope rupture and repair during cancer cell migration. Science 352, 353-358 
178 Raab, M., et al. (2016) ESCRT III repairs nuclear envelope ruptures during cell migration to limit DNA damage and cell death. Science 352, 359-362

179 Graham, D.M., et al. (2018) Enucleated cells reveal differential roles of the nucleus in cell migration, polarity, and mechanotransduction. J. Cell Biol. 217, 895-914

180 Gulvady, A.C., et al. (2018) Hic-5 expression is a major indicator of cancer cell morphology, migration, and plasticity in three-dimensional matrices. Mol. Biol. Cell 29, 1704-1717

181 Zhang, J. and Wang, Y.-I. (2017) Centrosome defines the rear of cells during mesenchymal migration. Mol. Biol. Cell 28, 3240-3251

$182 \mathrm{Kopf}$, A., et al. (2020) Microtubules control cellular shape and coherence in amoeboid migrating cells. J. Cell Biol. 219 (6): e201907154

183 Petrie, R.J., et al. (2012) Nonpolarized signaling reveals two distinct modes of 3D cell migration. J. Cell Biol. 197, 439-455 


\section{Figure 1}

\section{Distribution of the polarised cytoskeletal networks during mesenchymal cell migration}

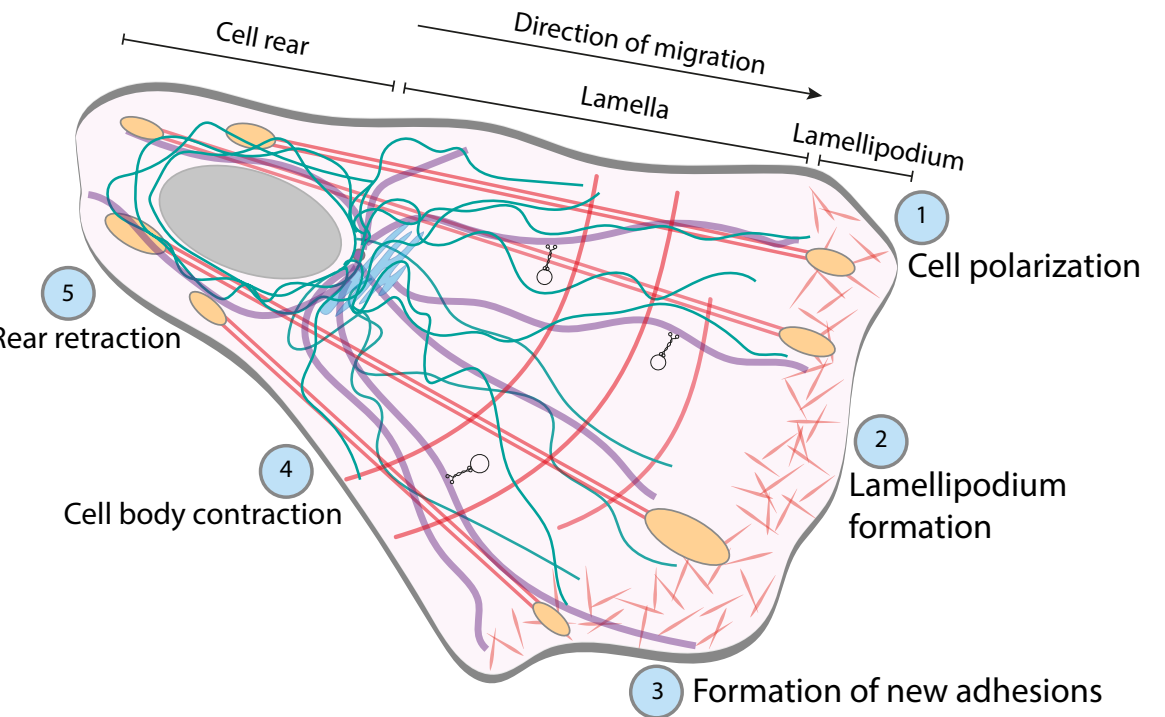

\begin{tabular}{|l|}
\hline$=$ Actomyosin stress fibre \\
Actin meshwork \\
Microtubule \\
Intermediate filament \\
Focal adhesion \\
MT-associated molecular motors \\
Vesicle \\
Nucleus \\
Golgi apparatus
\end{tabular}




\section{A. Cytoskeletal networks extend towards the cell periphery}

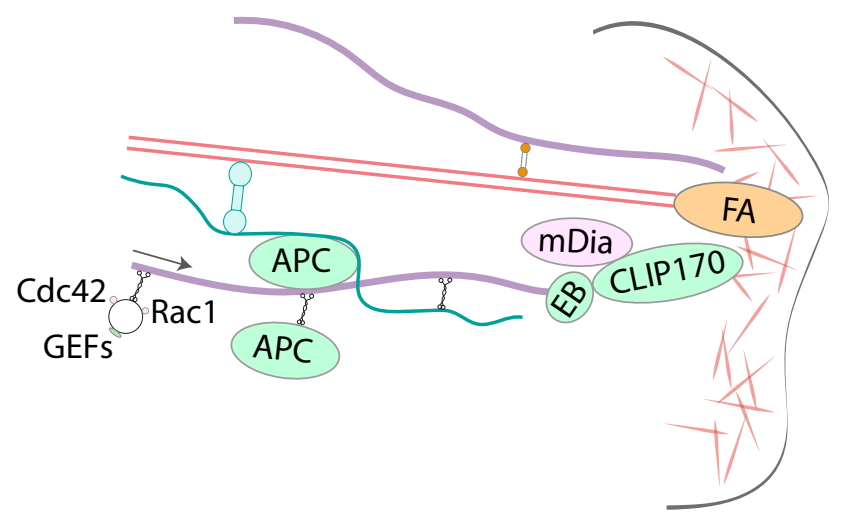

\section{B. Actin network as a physical barrier for MT growth}

MTs growing towards the cortex

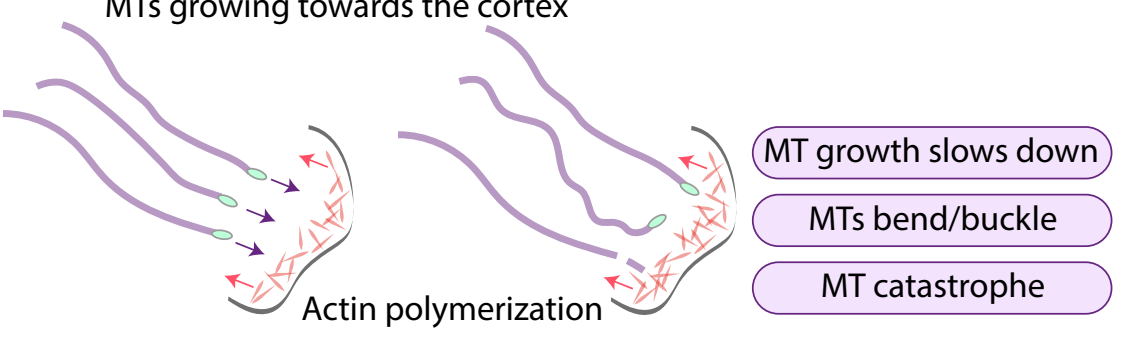

$$
\begin{aligned}
& \text { Actomyosin stress fibre } \\
& \text { Microtubule (MT) } \\
& \text { Intermediate filament (IF) } \\
& \text { Focal adhesion (FA) } \\
& \text { Actin meshwork } \\
& \text { MT-associated molecular motors } \\
& 0 \text { Vesicle } \\
& \therefore \text { Plectin } \\
& \therefore \text { ACF7 (Actin crosslinking factor } 7 \text { ) }
\end{aligned}
$$




\section{Regulation of FA turnover and contractility}

\section{A. MTs and actin}

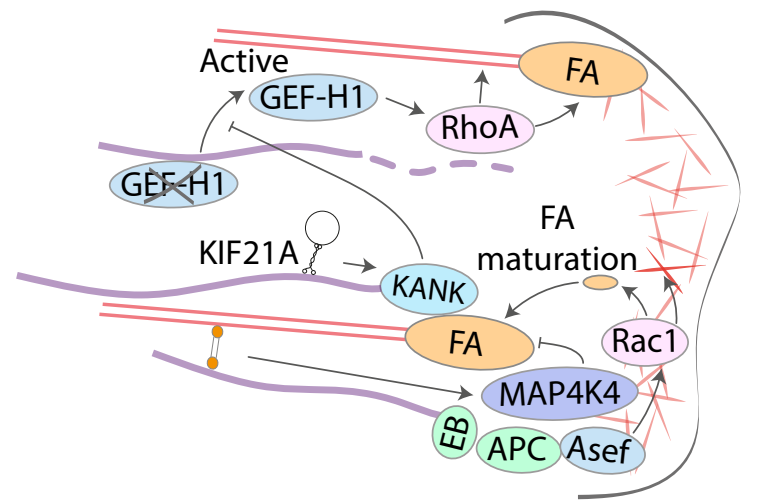

\section{B. IFs and actin/MTs}

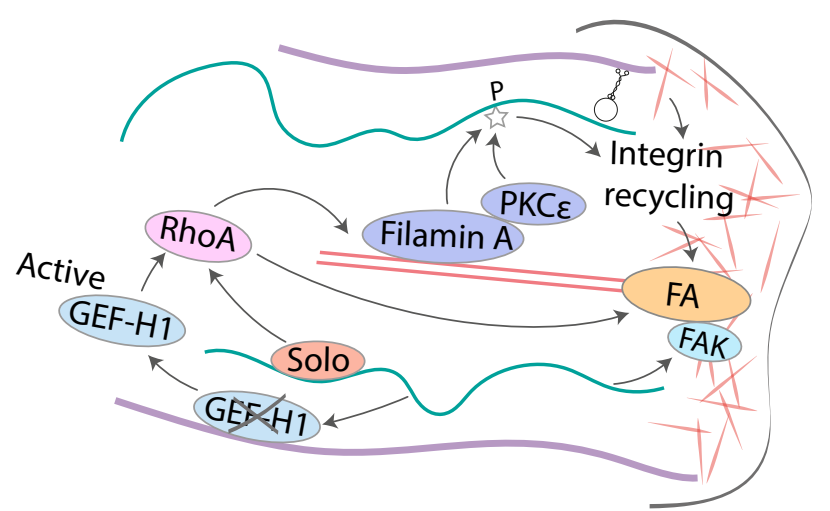




\section{Cytoskeletal crosstalk during cell migration}

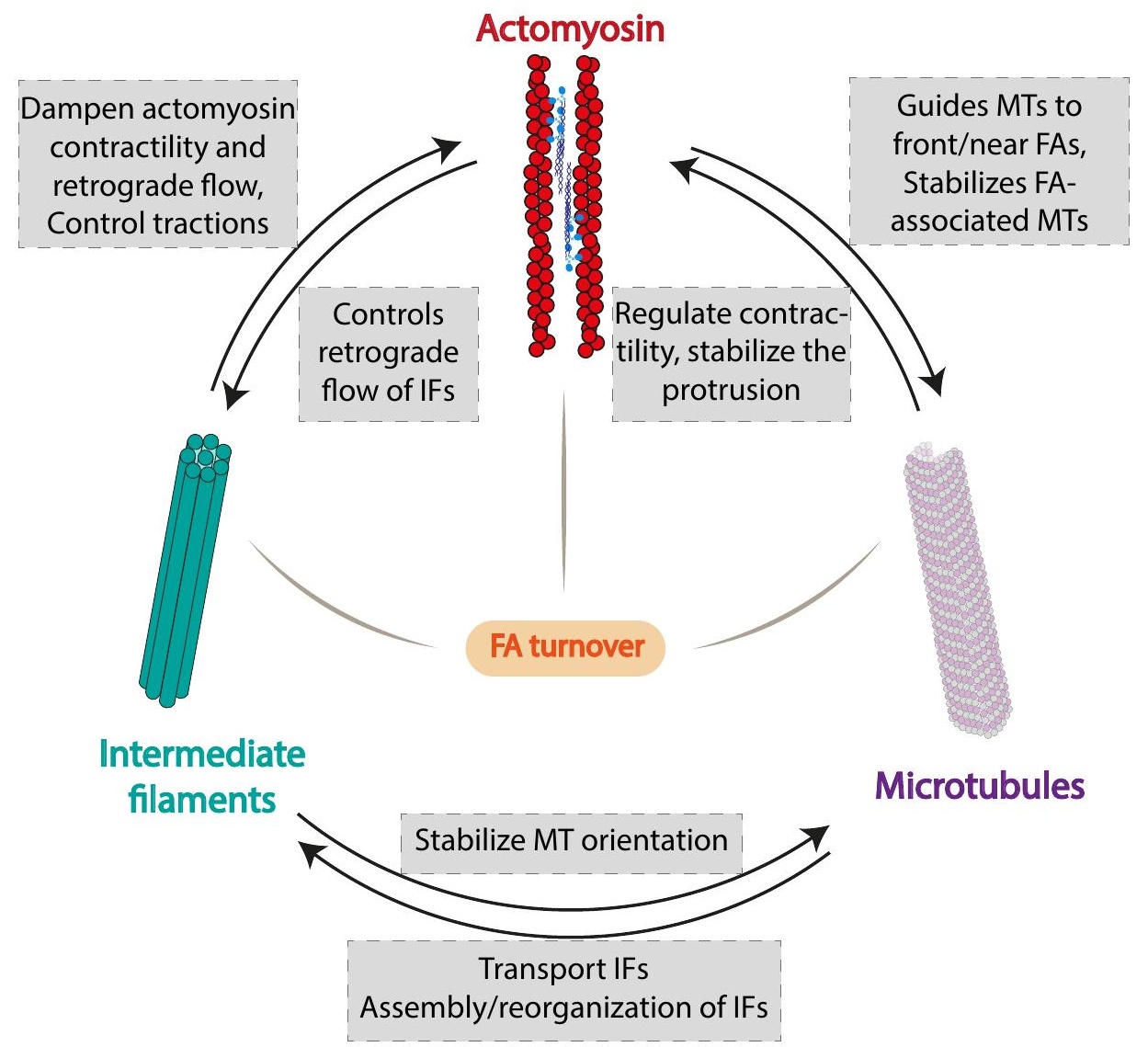


